\title{
Shallow donor in natural MoS2
}

\author{
Son Tien Nguyen, Yong-Sung Kim and Erik Janzén
}

\section{Linköping University Post Print}

N.B.: When citing this work, cite the original article.

Original Publication:

Son Tien Nguyen, Yong-Sung Kim and Erik Janzén, Shallow donor in natural MoS2, 2015, Physica Status Solidi. Rapid Research Letters, (9), 12, 707-710.

http://dx.doi.org/10.1002/pssr.201510297

Copyright: Wiley-VCH Verlag

http://www.wiley-vch.de/publish/en/

Postprint available at: Linköping University Electronic Press

http://urn.kb.se/resolve?urn=urn:nbn:se:liu:diva-123946 


\title{
Shallow donor in natural $\mathrm{MoS}_{2}$
}

\author{
Nguyen T. Son ${ }^{*, 1}$, Yong-Sung Kim ${ }^{* *, 2,3}$, and Erik Janzén ${ }^{1}$ \\ ${ }^{1}$ Department of Physics, Chemistry and Biology, Linköping University, SE-58183 Linköping \\ 2 Korea Research Institute of Standards and Science, Yuseong, Daejeon 305-340, Korea \\ ${ }^{3}$ Department of Nano Science, Korea University of Science and Technology, Daejeon 305-350, Korea
}

Received ZZZ, revised ZZZ, accepted ZZZ

Published online ZZZ (Dates will be provided by the publisher.)

Keywords shallow donor, valley-orbit splitting, electron paramagnetic resonance, density functional theory calculations.

* Corresponding author: e-mail: son@ifm.liu.se, Phone: +46 13 282531, Fax: +46 13137568

** e-mail: kimyongsung@gmail.com

Using electron paramagnetic resonance and density functional theory calculations, we show that the shallow donor responsible for the n-type conductivity in natural MoS2 is rhenium (Re) with a typical concentration in the low $10^{17}$ $\mathrm{cm}^{-3}$ range and the g-values: $\mathrm{g} \|=2.0274$ and $\mathrm{g}_{\perp}=2.2642$.
In bulk $\mathrm{MoS}_{2}$, the valley-orbit (VO) splitting and ionization energy of the Re shallow donor are determined to be $\sim 3$ and $\sim 26 \mathrm{meV}$, respectively. Calculations show that the VO splitting of Re approaches the value in bulk if the number of $\mathrm{MoS}_{2}$ layers is larger than four and increases to 97.9 $\mathrm{meV}$ in a monolayer.
Molybdenum disulphide $\left(\mathrm{MoS}_{2}\right)$ is an abundant mineral in the earth crust. In its layered structure, single layers of $\mathrm{MoS}_{2}$, each consisting of a sheet of Mo atoms sandwiched between two sheets of $\mathrm{S}$ atoms by strong covalent bonds $\mathrm{S}$ Mo-S, are stacked upon each other and held by weak van der Walls forces. In hexagonal structure $(2 \mathrm{H}), \mathrm{MoS}_{2}$ is a semiconductor with an indirect band gap of $~ 1.2 \mathrm{eV} \mathrm{[1][2].}$ Being the most common naturally-occurring polytype, $2 \mathrm{H}$ $\mathrm{MoS}_{2}$ is often referred to as $\mathrm{MoS}_{2}$. The fundamental properties [3][4] and photovoltaic effect [1][2][5][6] of the material have been studied for many years, but activities remains to be at a rather low level for several decades. Only recently, when advances in isolating single layers by exfoliation allowing detailed studies of two-dimensional material, the interest has been renewed. Starting with photoluminescence (PL) studies by Mak and co-workers [7], showing the transformation from an indirect band gap of $\sim 1.2 \mathrm{eV}$ in bulk to a direct band gap of $\sim 1.9 \mathrm{eV}$ in a monolayer, which exhibits an increase in luminescence quantum efficiency by a factor of more than $10^{4}$ compared with the bulk material. It has been followed by the successful fabrication of singlelayer $\mathrm{MoS}_{2}$ transistors with the electron mobility of $\sim 200$ $\mathrm{cm}^{2} / \mathrm{Vs}$, room-temperature current on/off ratios of $10^{8}$ and ultralow standby power dissipation [8] and of 10-nm thick
$\mathrm{MoS}_{2}$ transistors with the electron mobility as high as $\sim 700$ $\mathrm{cm}^{2} / \mathrm{Vs}$ at $295 \mathrm{~K}$ [9], showing advantages of the material in electronics and optoelectronics compared to graphene-the most studied two-dimensional material. Impressive integrated circuits [10][11], phototransistors [12] and sensors [13] based on single- and bi-layers of $\mathrm{MoS}_{2}$ have recently been demonstrated.

In most of the reported devices or studies, $\mathrm{MoS}_{2}$ layers exfoliated from natural bulk materials show $n$-type conductivity, but the origin of the shallow donor-the source of free carriers-and its electronic structure are not known. Rhenium is commonly present in natural $\mathrm{MoS}_{2}$ with the concentration varying in the range of $10^{17}-10^{21} \mathrm{~cm}^{-3}$ [14] and expected to be a donor when substituting for Mo. Intentional doping of rhenium ( $\mathrm{Re}$ ) is known to increase the conductivity of $\mathrm{MoS}_{2}$, but for unknown reasons the activation energy of Re has not been determined [15]. Recent calculations suggest Re to be a donor with the lowest activation energy (not less than $0.2 \mathrm{eV}$ ) among impurities substituting for Mo in $\mathrm{MoS}_{2}$ monolayer [16]. Information on the electronic structure of a shallow donors, which can usually be obtained from optical studies such as far-infrared absorption or PL, is lacking for $\mathrm{MoS}_{2}$. Reported PL spectra in single or few layers of $\mathrm{MoS}_{2}$ show only single peaks related to direct-

* Corresponding author: e-mail son@ifm.liu.se, Phone: +46 13 282531, Fax: +46 13137568 
and indirect-gap transitions without structure, while for unknown reasons PL emissions are either absent or negligible in bulk materials [7]. Identifying the donor responsible for the $n$-type conductivity of natural $\mathrm{MoS}_{2}$ and understanding its electronic structure are of fundamental defect physics and technological interests.

In this Letter, we show from our electron paramagnetic resonance (EPR) studies that the shallow donor in natural $\mathrm{MoS}_{2}$ bulk has a donor ionization energy of $\sim 27 \mathrm{meV}$ and a valley-orbit (VO) splitting of $\sim 3 \mathrm{meV}$. Calculations for all three possible donors substituting for Mo showed that when the number of layers exceeding four the VO splitting approaches the bulk values: 2.5, 6.8 and $50 \mathrm{meV}$ for Re, technetium (Tc) and manganese (Mn), respectively, suggesting that the shallow donor observed by EPR is Re. The VO splitting for Re is found to be increased to $97.9 \mathrm{meV}$ in a sing layer of $\mathrm{MoS}_{2}$.

Samples used in this study are bulk $\mathrm{MoS}_{2}$ from SPI. EPR measurements were performed on an X-band ( 9.42 $\mathrm{GHz}$ ) E500 spectrometer from Bruker equipped with a Heflow cryostat which allows the regulation of the sample temperature in the range of 4-295 K. In EPR measurements, the sample was kept in an open-surface plastic pocket without using any glue in order to avoid strain at low temperatures.

Figures 1(a) and 1(b) shows EPR spectra of $\mathrm{MoS}_{2}$ measured at $25 \mathrm{~K}$ for the magnetic field parallel $(\mathbf{B} \| \mathbf{c})$ and perpendicular $(\mathbf{B} \perp \mathbf{c})$ to the $c$ axis of the hexagonal lattice, respectively. The spectrum consists of a single line without any resolved hyperfine structure. The line width is $\sim 6.5 \mathrm{G}$ for $\mathbf{B} \| \mathbf{c}$ and becomes much narrower for $\mathbf{B} \perp \mathbf{c}(\sim 1.4 \mathrm{G})$. The angular dependence study of the EPR signal shows that the line does not split when rotating the magnetic field away from the $c$ axis, indicating an effective electron spin $S=1 / 2$ and $C_{3 \mathrm{v}}$ symmetry with the g-values: $\mathrm{g}_{\|}=2.0274$ and $\mathrm{g}_{\perp}=2.2642$ (at $25 \mathrm{~K}$ ). The lack of any hyperfine structure is an indication that the EPR center may be a shallow defect. (A deep level defect often shows strong ligand or self-hyperfine interactions due to its localized wave function.)

EPR spectra measured for $\mathbf{B} \perp \mathbf{c}$ at different temperatures are shown in Fig. 2(a). The signal is strongest at the lowest temperature and is drastically decreased with increasing temperature to $\sim 30 \mathrm{~K}$. At higher temperatures, the signal continues to decrease but with a much slower pace to a noise level at $\sim 150 \mathrm{~K}$. As can be seen in the figure, with increasing temperature, the resonance position shifts to higher magnetic fields corresponding to a decrease in the g-value from $2.2643(8)$ at $7 \mathrm{~K}$ to $2.2635(6)$ at $135 \mathrm{~K}$. The g-value of a shallow donor is known to be similar to that of free electrons [17] and is dependent on the band gap [18], e.g., decreasing with increasing temperature, that reduces the band gap, as observed for the Si shallow donor in AlN [19]. Such a temperature dependence of the EPR intensity is another evidence indicating that the center is a shallow donor.

Using spin-counting function integrated in the E500 spectrometer, we deduce the concentration of this donor at different temperatures from the integrated intensity of the EPR signal with taking into account the Boltzmann factor

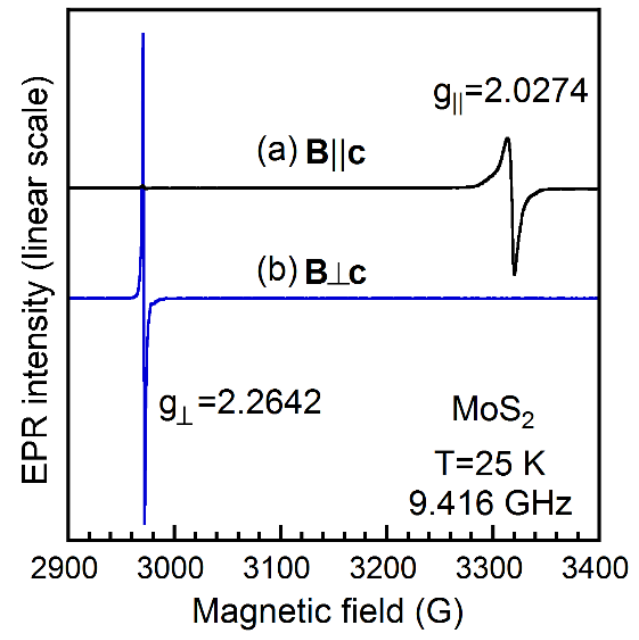

Figure 1 (Color online) EPR spectra in $2 \mathrm{H}-\mathrm{MoS}_{2}$ measured at $25 \mathrm{~K}$ for (a) $\mathbf{B} \| \mathbf{c}$ and (b) $\mathbf{B} \perp \mathbf{c}$. The microwave power and the field modulation are: $2 \mathrm{~mW}$ and $0.8 \mathrm{G}$ for (a) and $0.06325 \mathrm{~mW}$ and $0.3 \mathrm{G}$ for (b). The microwave frequency is calibrated to $9.416 \mathrm{GHz}$.

that causes the difference in population between the two Zeeman splitting levels $\left(M_{S}= \pm 1 / 2\right)$. The results are plotted in Fig. 2(b) as open circles. We found that the concentration is highest at the lowest temperature $\left(\sim 3.5 \times 10^{17} \mathrm{~cm}^{-3}\right.$ at 10 $\mathrm{K})$ and rapidly decreases with increasing temperature to $\sim 30$ $\mathrm{K}$ and then gradually reduces at higher temperatures. Considering the case of an effective-mass donor, its concentration on the ground state $1 s$ (or $\left.E_{d}\right), n(T)$, can be described by the Boltzmann distribution

$$
\mathrm{n}(\mathrm{T}) \propto \frac{\mathrm{N} 2 \mathrm{e}^{-\left(\mathrm{E}_{\mathrm{d}}-\mathrm{E}_{\mathrm{F}}\right) / \mathrm{kT}}}{1+2 \mathrm{e}^{-\left(\mathrm{E}_{\mathrm{d}}-\mathrm{E}_{\mathrm{F}}\right) / \mathrm{k}_{\mathrm{B}} \mathrm{T}}+\sum_{\mathrm{i}} \mathrm{e}^{-\left(\mathrm{E}_{\mathrm{i}}-\mathrm{E}_{\mathrm{F}}\right) / \mathrm{k}_{\mathrm{B}} \mathrm{T}} .}
$$

Here, $\mathrm{N}$ is the total donor concentration, $\mathrm{E}_{\mathrm{F}}$ is the Fermi level, $E_{i}$ is the energy of excited states of the donor, $k_{B}$ is the Boltzmann constant and the energy of the conduction band mimnium is set to zero $\left(E_{C}=0\right)$. We also assume that no free electrons in the conduction band and no double occupation of a single level. Under the external magnetic field, the donor ground state is split into two spin states $\left(M_{S}= \pm 1 / 2\right)$, each can be occupied by one electron. Neclecting the difference in energy of these spins states, we have two states with energy $E_{\mathrm{d}}$. Details on Boltzmann distribution are described in Supplementary Information. For bulk $\mathrm{MoS}_{2}$ with an indirect band gap, there are six equivalent conduction band minima (CBM) located in between $K$ and $\Gamma$ points of the Brillouin zone. In pristine $\mathrm{MoS}_{2}$, the six states related to the six equivalent CBM are degenerate. In the presence of a shallow donor, the valley-orbit interaction splits the ground state of the donor into four states: a singlet ground state $\left(a_{1}\right)$, two doublet $\left(e, e^{*}\right)$ and another singlet $\left(a_{1} *\right)$ state with $e$ being closest to the ground state $a_{1}$. The energy separation $e-a_{1}$ is 
often called the VO splitting. The VO splitting is often smaller than the energy distance between the ground state $1 s\left(\mathrm{E}_{\mathrm{d}}\right)$ and the first excited state $2 p$ in known semiconductors, e.g. $\mathrm{Si}, \mathrm{Ge}$ [20] and SiC [21]. Thus, at low temperatures, the decrease of $n(T)$ is mainly due to the removal of electrons from the ground state $E_{d}$ to the $E_{v o}$ state by thermal energy and the influence from higher excited states can be neglected. The Eq. (1) then can be written as

$$
\mathrm{n}(\mathrm{T}) \propto \frac{\mathrm{N}}{1+0.5 \mathrm{e}^{\left(\mathrm{E}_{\mathrm{d}}-\mathrm{E}_{\mathrm{F}}\right) / \mathrm{k}_{\mathrm{B}} \mathrm{T}}+0.5 \mathrm{~g}_{1} \mathrm{e}^{-\left(\mathrm{E}_{\mathrm{VO}}-\mathrm{E}_{\mathrm{d}}\right) / \mathrm{k}_{\mathrm{B}} \mathrm{T}} .}
$$

Here $g_{1}$ is the degenerate factor of the $\mathrm{E}_{\mathrm{vo}}$ state. Fitting the temperature dependence of $n(T)$ at low temperatures considering only the ground state $\mathrm{E}_{\mathrm{d}}$ and the first excited state $E_{V O}$ using Eq. (2), we observed: $E_{d}-E_{F}=-2.6 m e V, E_{V O}-E_{d}$ $=2.66 \sim 3 \mathrm{meV}$, and $\mathrm{N}=2.97 \times 10^{17} \sim 3 \times 10^{17} \mathrm{~cm}^{-3}$.

With increasing further the temperature, electrons from the $\mathrm{E}_{\mathrm{Vo}}$ and $\mathrm{E}_{\mathrm{d}}$ states can be excited to the $2 p$ excited state. This process leads to a decrease of $\mathrm{n}(\mathrm{T})$ as shown in the temperature range of $\sim 40-135 \mathrm{~K}$ in Fig. 2(b). The change of $n(T)$ in this temperature range depends mainly on the energy distance between the ground state $\mathrm{E}_{\mathrm{d}}$ and the $2 p$ state, $\mathrm{E}_{2 p}-\mathrm{E}_{\mathrm{d}}$. Once electrons can be excited to the $2 p$ state, they can move up to higher excited states within $\mathrm{k}_{\mathrm{B}} \mathrm{T}$. Therefore, we make an approximation, considering only the ground state and the $2 p$ state while the population on higher excited states is accounted for by the degenerate factor $C$. The variation of the donor concentration $n(T)$ in this elevated temperature range then can be described as

$$
\mathrm{n}(\mathrm{T}) \propto \frac{\mathrm{N}}{1+0.5 \mathrm{e}^{\left(\mathrm{E}_{\mathrm{d}}-\mathrm{E}_{\mathrm{F}}\right) / \mathrm{k}_{\mathrm{B}} \mathrm{T}}+\mathrm{Ce}^{-\left(\mathrm{E}_{2 \mathrm{p}}-\mathrm{E}_{\mathrm{d}}\right) / \mathrm{k}_{\mathrm{B}} \mathrm{T}} .}
$$

Here the factor $\mathrm{C}$ is the degenerate factor of all the excited states within $\mathrm{k}_{\mathrm{B}} \mathrm{T}$ from the $2 p$ state. Fitting the data in the temperature range 40-135 K using Eq. (3), we obtained: $E_{d^{-}}$ $\mathrm{E}_{\mathrm{F}}=-3.81 \mathrm{meV}, \mathrm{E}_{2 p}-\mathrm{E}_{\mathrm{d}}=20.54 \mathrm{meV}, \mathrm{C}=18$ and $\mathrm{N}=$ $8.3 \times 10^{16} \mathrm{~cm}^{-3}$. Assuming that the neutral state $E_{d}$ and excited states of the donor follow the effective mass theory (EMT), i.e., the $\mathrm{E}_{\mathrm{d}} / \mathrm{i}^{2}$ rule $(\mathrm{i}=1,2, . ., \mathrm{n})$ or $\mathrm{E}_{2 p} \sim \mathrm{E}_{\mathrm{d}} / 4$ and $\mid \mathrm{E}_{2 p^{-}}$ $\mathrm{E}_{\mathrm{d}}|=|\left(\mathrm{E}_{\mathrm{d}} / 4\right)-\mathrm{E}_{\mathrm{d}}|=3| \mathrm{E}_{\mathrm{d}} \mid / 4$, we can estimate $\left|\mathrm{E}_{\mathrm{d}}\right|$ as: $\left|\mathrm{E}_{\mathrm{d}}\right|=$ $4\left|\mathrm{E}_{2 p}-\mathrm{E}_{\mathrm{d}}\right| / 3=27.4 \sim 27 \mathrm{meV}$. Thus, the observed shallow donor has a VO splitting of $\sim 3 \mathrm{meV}$ and an ionization energy $\mathrm{E}_{\mathrm{d}} \sim 27 \mathrm{meV}$. The simulations using the parameters obtained from the fits for low and elevated temperature ranges are plotted in Fig. 2(b) as solid curves. It should be noticed that the EMT may not be applicable in two-dimensional materials when one of the effective mass component along the direction perpendicular to the layer approaches infinity [22].

Transition metal elements in column VIIB (Mn, Tc and $\mathrm{Re}$ ) substituting for Mo are donors in $\mathrm{MoS}_{2}$. In order to find out the best candidate for the observed shallow donor, we calculate their VO splitting in $\mathrm{MoS}_{2}$. Density-functional theory (DFT) calculations are performed as implemented in Vienna Ab initio Simulation Package (VASP) code [23,24].
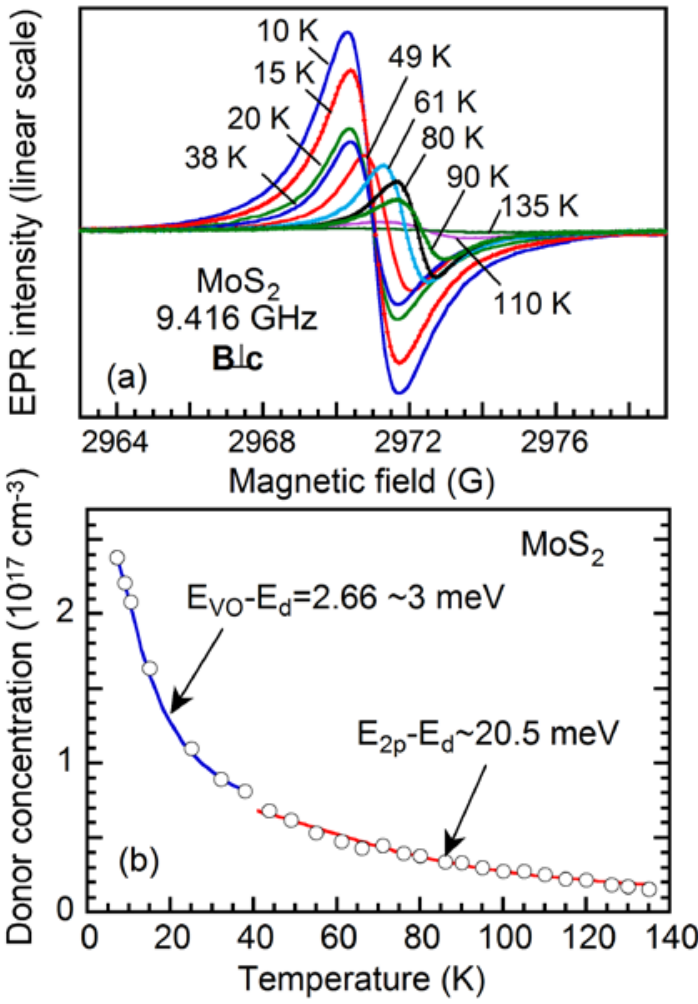

Figure 2 (Color online) (a) Temperature dependence of the EPR spectrum of measured for $\mathbf{B} \perp \mathbf{c}$ in the range of $10-135 \mathrm{~K}$ with a field modulation of $0.3 \mathrm{G}$ and a low microwave power of $0.02 \mathrm{~mW}$ which is far below the saturation level. The shift of the resonance line to higher magnetic fields is typical for a shallow donor whose g-value decreases with increasing temperature. (b) Temperature dependence of the donor concentration $\mathrm{n}(\mathrm{T})$ on the ground state level $1 \mathrm{~s}$ estimated from EPR (open circles) and the simulations (solid curves) using the parameters obtained from the fits for low and elevated temperature ranges using Eq. (2) and Eq. (3), respectively.

The ultrasoft pseudopotentials [25] and the local densityfunctional approximation (LDA) [26] are used. A kinetic energy cutoff of $350 \mathrm{eV}, 6 \times 6 \times 32 \mathrm{H}-\mathrm{MoS}_{2}$ supercell (648 host atoms), and $2 \times 2 \times 2 \Gamma$-centered k-point mesh for the supercell are used. Details of the DFT calculation of the VO splitting are described in Supplementary Information. The sixfold degeneracy of the CBM in pristine bulk $\mathrm{MoS}_{2}$ is found to split into a singlet ground state $\left(a_{1}\right)$, a doublet state $(e)$, another doublet state $\left(e^{*}\right)$, and another singlet state $\left(a_{1} *\right)$ in increasing order of the energy level with the presence of Mo-substitutional Mn, Tc, and Re donor. The calculated VO split levels are listed in Table 1, and the VO splits between the $a_{1}$ and $e$ states for the $\mathrm{Mn}, \mathrm{Tc}$, and Re donors are found to be 50.0, 6.8, and $2.5 \mathrm{meV}$, respectively. The calculated VO splitting for Re is very close to the corresponding value determined by EPR, suggesting that the observed shallow donor in natural $\mathrm{MoS}_{2}$ is Rhenium. 
Table 1 Calculated six conduction VO split levels (in $\mathrm{meV}$ ) in pristine, Mn, Tc, and Re-doped bulk $\mathrm{MoS}_{2}$ are listed.

\begin{tabular}{ccccc}
\hline Level & Pristine & Mn & Tc & Re \\
\hline$a_{1}{ }^{*}(1)$ & 0 & 141.2 & 48 & 24.1 \\
$e^{*}(2)$ & 0 & 139.8 & 45.4 & 19.8 \\
$e(2)$ & 0 & 50 & 6.8 & 2.5 \\
$a_{1}(1)$ & 0 & 0 & 0 & 0 \\
\hline
\end{tabular}

Table 2 Calculated six conduction VO split levels (in meV) in Redoped bulk and few-layer (2-6 layer) $\mathrm{MoS}_{2}$, and two conduction VO split levels in Re-doped monolayer $\mathrm{MoS}_{2}$ are listed.

\begin{tabular}{ccccc}
\hline Level & $a_{1}{ }^{*}(1)$ & $e^{*}(2)$ & $e(2)$ & $a_{1}(1)$ \\
\hline Bulk & 24.1 & 19.8 & 2.5 & 0 \\
$6 \mathrm{~L}$ & 29.3 & 23.9 & 3.1 & 0 \\
$5 \mathrm{~L}$ & 30.8 & 25.0 & 2.9 & 0 \\
$4 \mathrm{~L}$ & 34.4 & 28.0 & 3.7 & 0 \\
3 L & 38.3 & 31.2 & 4.2 & 0 \\
2 L & 57.6 & 49.9 & 17.4 & 0 \\
$1 \mathrm{~L}$ & 97.9 & & & 0 \\
\hline
\end{tabular}

The VO splits in Re-doped monolayer and few-layer $\mathrm{MoS}_{2}$ are also investigated. For monolayer $\mathrm{MoS}_{2}$, the CBM is located at the $K$ valley in the Brillouin zone and thus twofold degenerated. With the presence of Re donor substituting Mo in monolayer $\mathrm{MoS}_{2}$, they split into a singlet ground state $\left(a_{1}\right)$ and a singlet excited state $\left(a_{1} *\right)$. For few-layer $\mathrm{MoS}_{2}$ thicker than 3-layer, the most stable layer of Mo-substitutional $\mathrm{Re}$ is found to be the central $\mathrm{MoS}_{2}$ layer rather than the surface $\mathrm{MoS}_{2}$ layer. The VO splits for the most stable Re are listed in Table 2. When the $\mathrm{MoS}_{2}$ is thicker than 4-layer, the VO split becomes closer to the value in bulk.

In summary, we have observed in natural $\mathrm{MoS}_{2}$ an EPR spectrum of a shallow donor with $C_{3 \mathrm{v}}$ symmetry and g-values: $g_{\|}=2.0274$ and $g_{\perp}=2.2642$ (at $25 \mathrm{~K}$ ). From the temperature dependence study of the donor concentration, a VO splitting of $\sim 3 \mathrm{meV}$ and an ionization energy of $\sim 27 \mathrm{meV}$ were determined for the shallow donor. The observed VO splitting is in good agreement with the calculated value of $2.5 \mathrm{meV}$ for Re in bulk $\mathrm{MoS}_{2}$, suggesting the shallow donor, that responsible for the $n$-type conductivity of natural $\mathrm{MoS}_{2}$, to be rhenium. The calculations show that the VO splitting approaches the value in bulk when the number of layers exceeding four and increases to $97.9 \mathrm{meV}$ in monolayers.

Acknowledgements Support from the Linköping Linnaeus Initiative for Novel Functional Materials (LiLi-NFM) is acknowledged. YSK acknowledges the support from Korea Evaluation Institute of Industrial Technology (KEIT) funded by the Ministry of Trade, Industry and Energy (MOTIE) (Project No. 10050296: Large scale (over 8”) synthesis and evaluation technology of 2-dimensional chalcogenides for next generation electronic devices).

\section{References}

[1] K. K. Kam and B. A. Parkinson, J. Phys. Chem. 86, 463 (1982).
[2] E. Fortin and W. M. Sears, J. Phys. Chem. Solids 43, 881 (1982).

[3] J. A. Wilson and A. D. Yoffe, Adv. Phys. 18, 193 (1969).

[4] L. F. Mattheiss, Phys. Rev. B 8, 3719 (1973).

[5] H. Tributsch, Sol. Energy Mater. 1, 257 (1979).

[6] L. F. Schneemeyer, M. S. Wrighton, A. Stacy, and M. J. Sienko, Appl. Phys. Lett. 36, 701 (1980).

[7] K. F. Mak, C. Lee, J. Hone, J. Shan, and T. F. Heinz, Phys. Rev. Lett. 105, 136805 (2010).

[8] B. Radisavljevic, A. Radenovic, J. Brivio, V. Giacometti, and A. Kis, Nat. Nanotechnol. 6, 147 (2011).

[9] S. Das, H.-Y. Chen, A. V. Penumatcha, and J. Appenzeller, Nano Lett. 13, 100 (2013).

[10] B. Radisavljevic, M. B. Whitwick, and A. Kis, ACS Nano 5, 9934 (2011).

[11] H. Wang, L. Yu, Y.-H. Lee, Y. Shi, A. Hsu, M. L. Chin, L.-J. Li, M. Dubey, J. Kong, and T. Palacios, Nano Lett. 12, 4674 (2012).

[12] Z. Yin, H. Li, H. Li, L. Jiang, Y. Shi, Y. Sun, G. Lu, Q. Zhang, X. Chen, and H. Zhang, ACS Nano 6, 74 (2012).

[13] H. Li, Z. Yin, Q. He, H. Li, X. Huang, G. Lu, D. W. H. Fam, A. I. Y. Tok, Q. Zhang, and H. Zhang, Small 8, 63 (2012).

[14] J. Golden, M. McMillan, R. T. Downs, G. Hystad, I. Goldstein, H. J. Stein, A. Zimmerman, D. A. Sverjensky, J. T. Armstrong, and R. M. Hazen, Earth Planet. Sci. Lett. 366, 1 (2013).

[15] K. K. Tiong, P. C. Liao, C. H. Ho, and Y. S. Huang, J. Cryst. Growth 205, 543 (1999).

[16] K. Dolui, I. Rungger, C. Das Pemmaraju, and S. Sanvito, Phys. Rev. B 88, 075420 (2013).

[17] C. F. Young, E. H. Poindexter, G. J. Gerardi, W. L. Warren, and D. J. Keeble, Phys. Rev. B 55, 16245 (1997).

[18] D. J. Chadi, A. H. Clark, and R. D. Burnham, Phys. Rev. B 13, 4466 (1976).

[19] N. T. Son, M. Bickermann, and E. Janzén, Appl. Phys. Lett. 98, 092104 (2011).

[20] A.K. Ramdas and S. Rodriguez, Rep. Prog. Phys. 44, 1297 (1981)

[21] I. G. Ivanov, A. Stelmach, M. Kleverman, and E. Janzén, Mater. Sci. Forum 483-485, 511 (2005).

[22] W. Kohn and J. M. Luttinger, Phys. Rev. 98, 915 (1955).

[23] G. Kresse and J. Furthmüller, Comput. Mater. Sci. 6, 15 (1996).

[24] G. Kresse and J. Furthmüller, Phys. Rev. B 54, 11169 (1996).

[25] D. Vanderbilt, Phys. Rev. B 41, 7892 (1990).

[26] D. M. Ceperley and B. J. Alder, Phys. Rev. Lett. 45, 566 (1980) 


\section{Supplementary Information}

\section{VO splitting and DFT calculation}

In semiconductors with the conduction band minimum (CBM) located at a low-symmetric k-point, there can be several equivalent CBMs. In pristine crystals, their electronic states are degenerated. In the presence of a donor impurity, its central core potential modifies the Coulomb potential which splits up the degeneracy of the CBMs and the energy states of the donor. The splitting depends on the central core potential and, hence, on the wave functional of the donor at the core, which is different for different orbitals of the donor electron. The interaction is therefore called valley-orbit (VO) interaction. The VO interaction is strong for the donors with the electron occupying s-orbitals with fully symmetric wave function and negligible for the donors on $p$ - and $d$-orbitals whose wave functions have a node at the core. Due to the VO interaction, the $1 \mathrm{~s}$ ground state of the donor is split up into several states depending on the symmetry of the CBM. The energy distance between the ground state and the first excited state of the $1 s$ manifold is called the VO splitting. The VO interaction is expected to be varied with impurities.

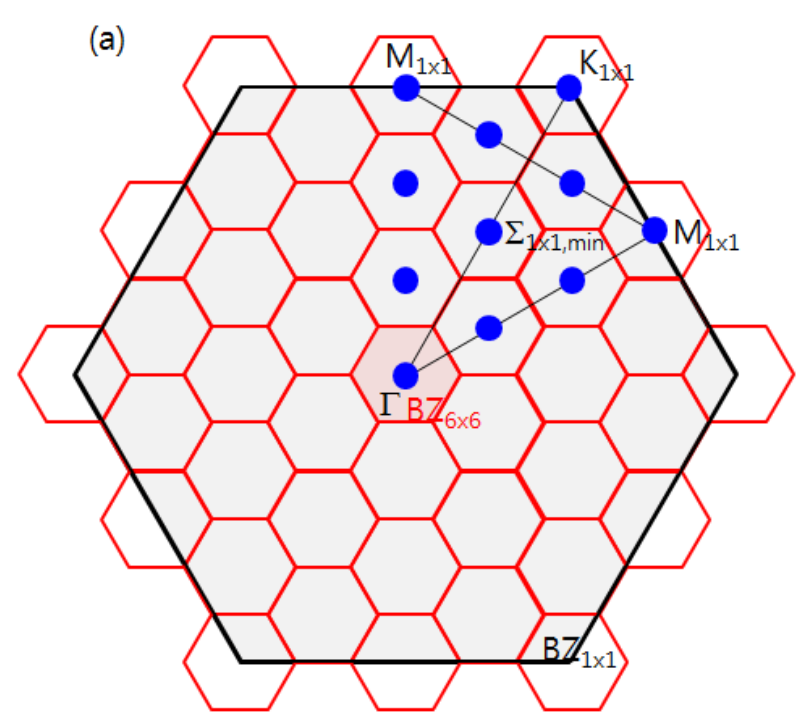

(b)

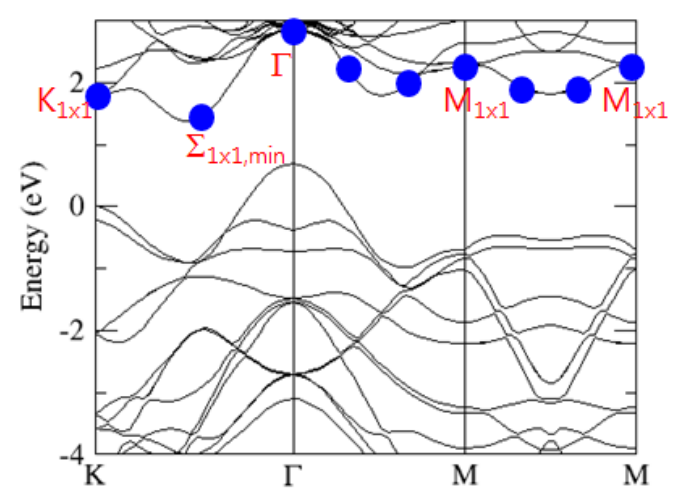

Fig. S1. (a) Hexagonal Brillioun zone (BZ) of the $1 \times 1$ unit cell $\left(\mathrm{BZ}_{1 \times 1}\right)$ (black) and that of the $6 \times 6$ supercell $\left(\mathrm{BZ}_{6 \times 6}\right)$ (red). (b) Band structure of bulk $\mathrm{MoS}_{2}$ in $\mathrm{BZ}_{1 \times 1}$. The lowest conduction states at the k-points that are folded onto the $\Gamma$ in $\mathrm{BZ}_{6 \times 6}$ are indicated by the blue circles in the $1 \times 1$ band structure.

In DFT calculations, a supercell is typically used for the study of a defect in a crystal. For Redoped $\mathrm{MoS}_{2}$, we can use a $6 \times 6$ supercell, and the Brillouin zone (BZ) of the $6 \times 6$ supercell (BZ $6 \times 6$ ) is plotted in Fig. S1(a) with the BZ of the $1 \times 1$ unit cell $\left(\mathrm{BZ}_{1 \times 1}\right)$. With this $6 \times 6$ supercell, the $\mathrm{k}$ points indicated by the blue circles are folded onto $\Gamma$ in $\mathrm{BZ}_{6 \times 6}$. The $\Gamma$ in $\mathrm{BZ}_{6 \times 6}$ then includes the kpoints, such as $\Gamma, \mathrm{M}$, and $\mathrm{K}$ in $\mathrm{BZ}_{1 \times 1}$. The energy levels of the lowest conduction states at the $\mathrm{k}$ points are indicated by the blue circles in the $1 \times 1$ band structure in Fig. S1(b). The conduction band minimum (CBM) in bulk $\mathrm{MoS}_{2}$ is located at a point along the $\sum$-line connecting $\Gamma$ and $\mathrm{K}$ in $\mathrm{BZ}_{1 \times 1}$, 


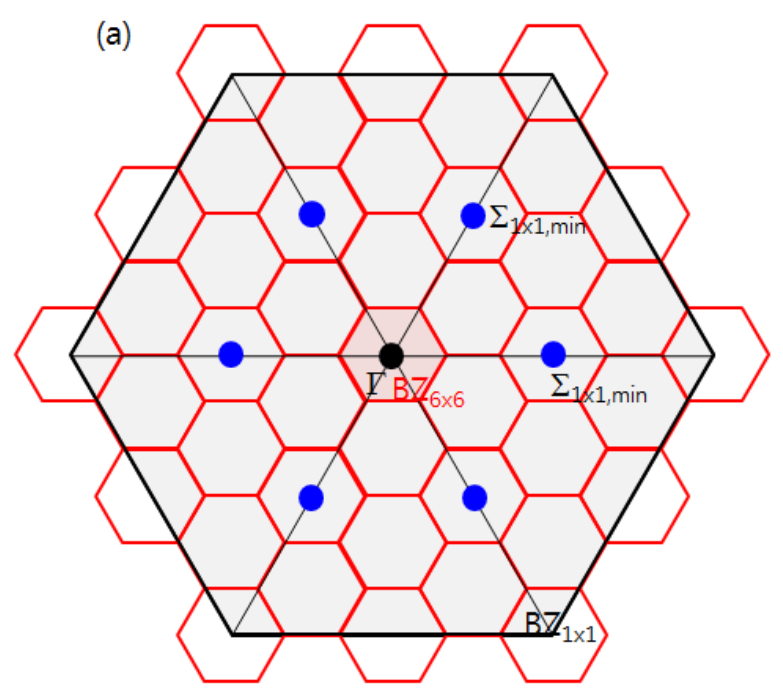

(b)

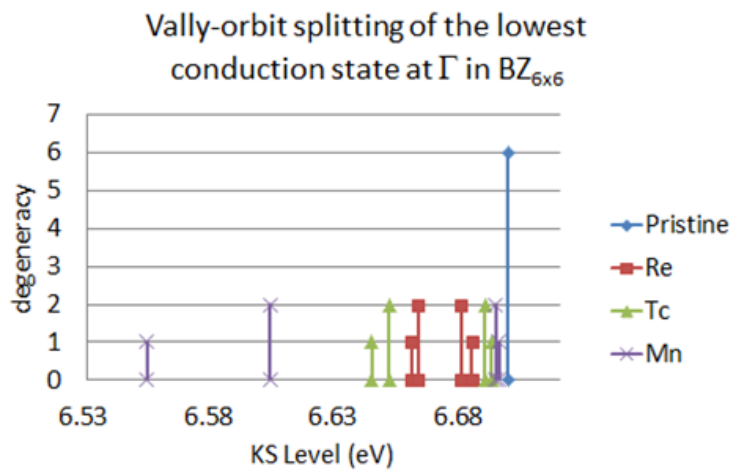

Fig. S2. (a) Hexagonal Brillioun zone (BZ) of the $1 \times 1$ unit cell $\left(\mathrm{BZ}_{1 \times 1}\right)$ (black) and that of the $6 \times 6$ supercell $\left(\mathrm{BZ}_{6 \times 6}\right)$ (red). The six-fold degenerated $\sum_{1 \times 1 \text {,min }}$ states are indicated by the blue circles. (b) The KS energy levels and the degeneracies of the lowest six conduction states at $\Gamma$ in $\mathrm{BZ}_{6 \times 6}$, which are equivalent to the $\sum_{1 \times 1 \text {,min }}$ states in $\mathrm{BZ}_{1 \times 1}$, are shown for pristine, Mo-substitutional Re, Tc, and Mn doped bulk $\mathrm{MoS}_{2}$.

and we call this point $\sum_{1 \times 1 \text {,min. }}$ The CBM at the $\sum_{1 \times 1 \text {,min }}$ is lower in energy level by about $0.2 \mathrm{eV}$ than the other energy levels at the k-points, and thus the CBM at the $\sum_{1 \times 1, \text { min }}$ is clearly distinguished from the other states at the $\Gamma$ point in $\mathrm{BZ}_{6 \times 6}$.

The lowest CBM at the $\sum_{1 x 1, \text { min }}$ [the blue circles in Fig. S2(a)] is six-fold degenerated, and they are fold onto $\Gamma$ in $\mathrm{BZ}_{6 \times 6}$. The six-fold degeneracy (g) of the lowest $\mathrm{CBM}$ in pristine bulk $\mathrm{MoS}_{2}$ is thus found at the $\Gamma$ point in the $\mathrm{BZ}_{6 \times 6}$, and is indicated in the Fig. S2(b) by the blue circles. With a Mo-substitutional defect, the $1 \times 1$ periodicity of $\mathrm{MoS}_{2}$ crystal is not valid, and correspondingly the six-fold degeneracy of the CBM is broken. The calculated Kohn-Sham (KS) levels and the degeneracies of the levels with the defects are plotted in Fig. S2(b). We simply use the DFT KS levels (the absolute values are not meaningful, and here, we focus only on the degeneracies and the splitting). As shown in Fig. S2(b), the six-fold degeneracy of the CBM of pristine $\mathrm{MoS}_{2}$ is split with the presence of the defect. The splitting is different for different impurities. With this approach, we can calculate the valley-orbit (VO) splitting by a defect in a crystal in DFT supercell approach.

In order to assess the accuracy of the DFT calculated VO split, we test convergence with supercell sizes, different pseudopotentials, and exchange-correlations, for Re-doped bulk $\mathrm{MoS}_{2}$, and Fig. S3 shows the results. The convergence with respect to the supercell sizes is found to be achieved with the $6 \times 6 \times 2$ supercell within $1.3 \mathrm{meV}$. We use the $6 \times 6 \times 3$ supercell in this study, and thus the error due to the supercell size is expected to be less than $1.3 \mathrm{meV}$. 
(a)

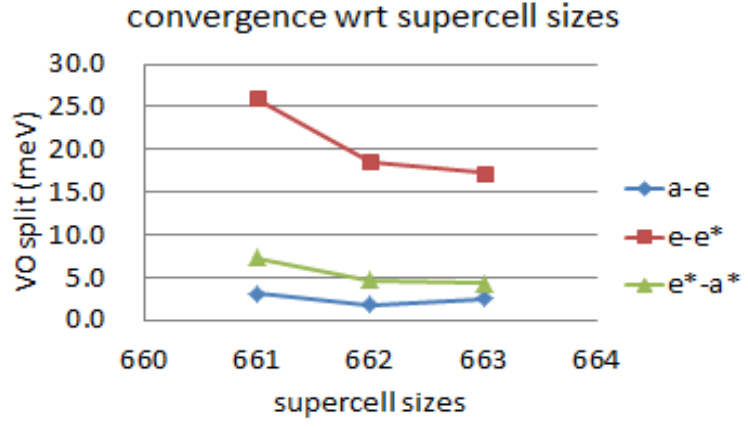

(b)

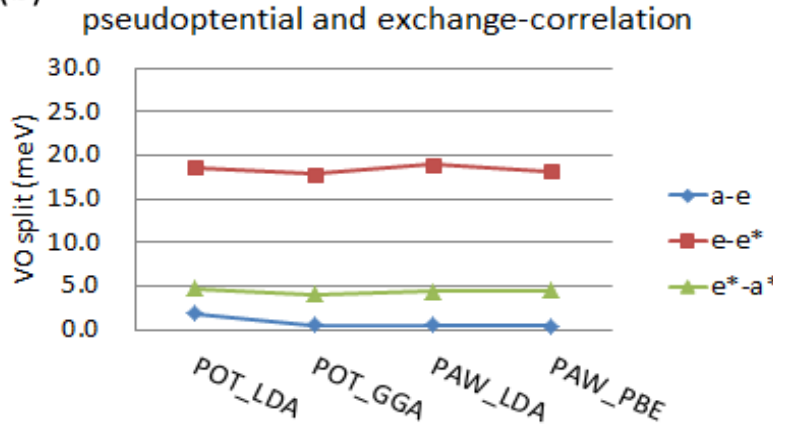

Fig. S3. (a) Calculated VO splits for Re-doped bulk $\mathrm{MoS}_{2}$ with $6 \times 6 \times 1$ (661), 6×6×2 (662), and 6×6×3 (663) supercells. (b) Calculated VO splits for Re-doped bulk $\mathrm{MoS}_{2}$ with $6 \times 6 \times 2$ supercell with different pseudopotentials and exchange-correlations.

We also test different exchange correlations and pseudopotentials, for Re-doped bulk $\mathrm{MoS}_{2}$ with the $6 \times 6 \times 2$ supercell, as shown in Fig. S3(b). When we use generalized-gradient approximation (GGA) with the ultra-soft pseudopotentials, the VO split values change by $1.3 \mathrm{meV}$ in maximum. When we use the plane-wave-augmented (PAW) pseudopotentials, the VO split values are found to be changed by $1.3 \mathrm{meV}$ in maximum. When we use the PAW pseudopotentials and GGA exchange-correlation, the VO split values are changed by $1.4 \mathrm{meV}$ in maximum. Based on these results, we assess the accuracy of the VO split calculation in our DFT study, and the error due to the choices of pseudopotentials and exchange-correlations can be about $1.4 \mathrm{meV}$.

The squared wave functions of the Re shallow donor states in bulk MoS2 are shown in Fig. S4(b)-(e). For comparison, the six-fold degenerated $\sum 1 \times 1$, min state in pristine bulk MoS2 is also shown in Fig. S4(a).

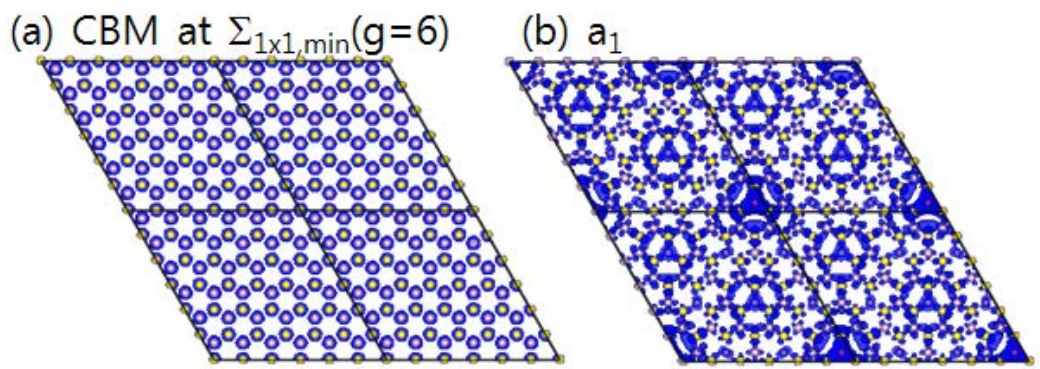

(c) e

(d) $e^{*}$

(e) $a_{1}{ }^{*}$

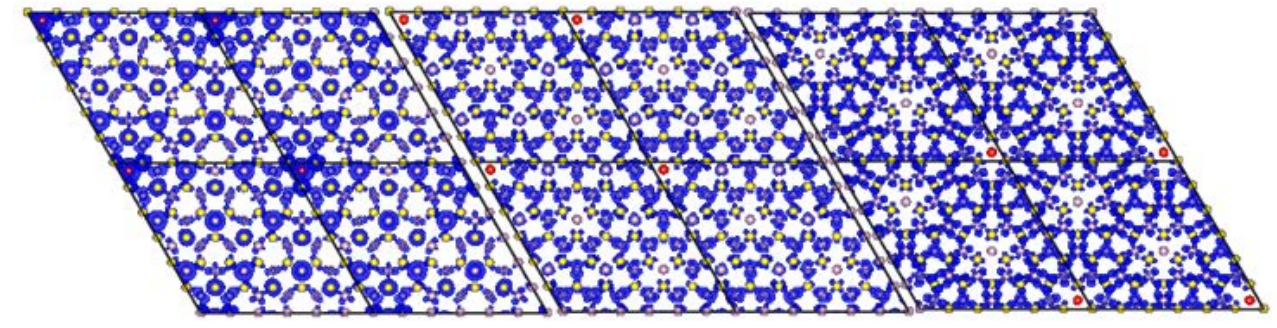

Fig. S4. (a) Six-fold degenerated CBM state at $\sum_{1 \times 1, \min }$ in pristine bulk $\mathrm{MoS}_{2}$. The VO-split (b) $\mathrm{a}_{1}$, (c) e, (d) e*, and (e) $\mathrm{a}_{1}$ * states in Re-doped bulk $\mathrm{MoS}_{2}$ are shown. Re is indicated by the red dots. 

(a) $\mathrm{CBM}$ at $\mathrm{K}_{1 \times 1}(\mathrm{~g}=2)$
(b) $a_{1}$
(c) $a_{1}{ }^{*}$

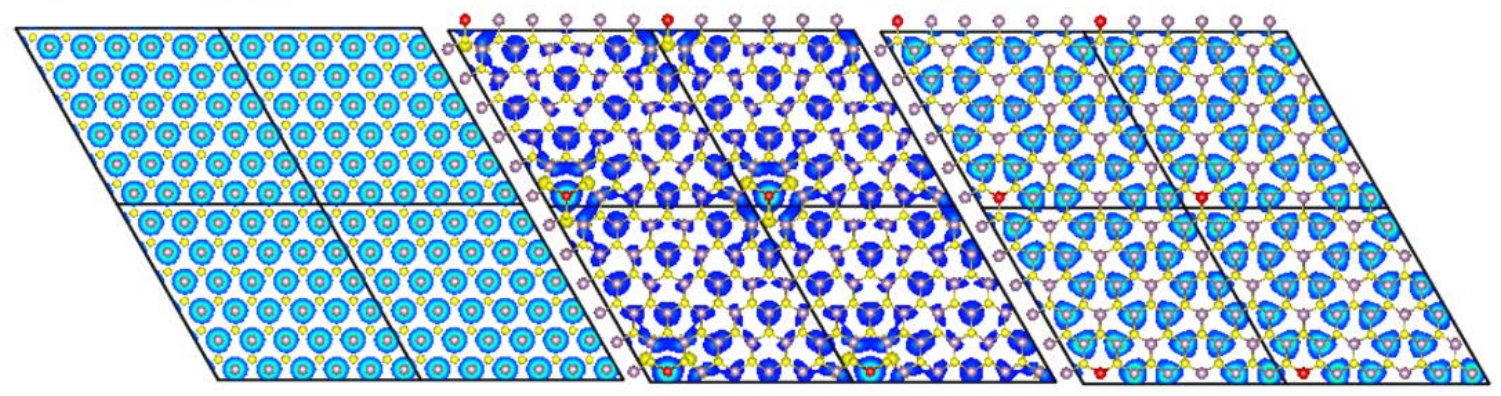

Fig. S5. (a) Two-fold degenerated CBM state at $\mathrm{K}_{1 \times 1}$ in pristine monolayer $\mathrm{MoS}_{2}$. The VO-split (b) $\mathrm{a}_{1}$ and (c) $\mathrm{a}_{1}{ }^{*}$ states in Re-doped monolayer $\mathrm{MoS}_{2}$ are shown. Re is indicated by the red dots.

The squared wave functions of the Re shallow donor states in monolayer $\mathrm{MoS}_{2}$ are shown in Fig. S5(b) and (c). For comparison, the two-fold degenerated $\mathrm{K}_{1 \mathrm{x} 1}$ state in pristine monolayer $\mathrm{MoS}_{2}$ is also shown in Fig. S5(a).

2. Boltzmann distribution and the concentration of the donor on the ground state

The average concentration of the donor on the ground state $E_{d}$ can be written as [1]:

$$
\mathrm{n}(\mathrm{T}) \propto \frac{\mathrm{Ng}_{\mathrm{d}} \mathrm{e}^{-\left(\mathrm{E}_{\mathrm{d}}-\mathrm{E}_{\mathrm{F}} \mathrm{g}_{\mathrm{d}}\right) / \mathrm{kT}}}{\mathrm{e}^{-\left(\mathrm{E}_{\mathrm{d}}-\mathrm{E}_{\mathrm{F}} \mathrm{g}_{\mathrm{d}}\right) / \mathrm{k}_{\mathrm{B}} \mathrm{T}}+\sum_{\mathrm{i}} \mathrm{e}^{-\left(\mathrm{E}_{\mathrm{i}}-\mathrm{E}_{\mathrm{F}} \mathrm{g}_{\mathrm{i}}\right) / \mathrm{k}_{\mathrm{B}} \mathrm{T}}+\mathrm{e}^{-\left(\mathrm{E}_{\mathrm{C}}-\mathrm{E}_{\mathrm{F}} \mathrm{g}_{\mathrm{CB}}\right) / \mathrm{k}_{\mathrm{B}} \mathrm{T}}}
$$

Here $\mathrm{N}$ is the total concentration of the donor, $\mathrm{g}_{\mathrm{d}}, \mathrm{g}_{\mathrm{i}}$ and $\mathrm{g}_{\mathrm{CB}}$ are the number of electrons on the corresponding states: ground state, excited states and the conduction band, $\mathrm{E}_{\mathrm{F}}$ is the Fermi level and the energy of the conduction band minimum is chosen as the reference energy $\left(E_{C}=0\right)$. Since the Coulomb repulsion of two localized electrons raises the energy of the doubly occupied level so high that double occupation is essentially prohibited and a single level will be occupied by one electron, i.e., $g_{i}=1$. Under the external magnetic field, the donor state will be split into two spin states with $M_{S}= \pm 1 / 2$. Neglecting the difference in energy of these two spin states, $E_{d,(-1 / 2)}$ and

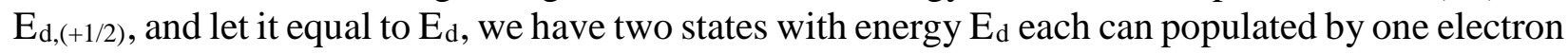
$\left(g_{d}=1\right)$ :

$$
\exp \left[-\left(E_{\mathrm{d},(-1 / 2)}-E_{F}\right) / k_{B} T\right]+\exp \left[-\left(E_{d,(+1 / 2)}-E_{F}\right) / k_{B} T\right]=2 \exp \left[-\left(E_{d}-E_{F}\right) / k_{B} T\right] .
$$

With measuring at low temperatures, we assume no electrons in the conduction band, i.e. $\mathrm{g}_{\mathrm{CB}}=0$. With $E_{C}=0$, thus: $\exp \left[-\left(E_{C}-E_{F} g_{C B}\right) / k_{B} T\right]=\exp \left(0 / k_{B} T\right)=1$. The $\operatorname{Eq}(1)$ then becomes:

$$
\mathrm{n}(\mathrm{T}) \propto \frac{\mathrm{N} 2 \mathrm{e}^{-\left(\mathrm{E}_{\mathrm{d}}-\mathrm{E}_{\mathrm{F}}\right) / \mathrm{kT}}}{2 \mathrm{e}^{-\left(\mathrm{E}_{\mathrm{d}}-\mathrm{E}_{\mathrm{F}}\right) / \mathrm{k}_{\mathrm{B}} \mathrm{T}}+\sum_{\mathrm{i}} \mathrm{e}^{-\left(\mathrm{E}_{\mathrm{i}}-\mathrm{E}_{\mathrm{F}}\right) / \mathrm{k}_{\mathrm{B}} \mathrm{T}}+1}
$$




$$
\mathrm{n}(\mathrm{T}) \propto \frac{\mathrm{N}}{1+0.5 \sum_{\mathrm{i}} \mathrm{e}^{-\left(\mathrm{E}_{\mathrm{i}}-\mathrm{E}_{\mathrm{d}}\right) / \mathrm{k}_{\mathrm{B}} \mathrm{T}}+0.5 \mathrm{e}^{\left(\mathrm{E}_{\mathrm{d}}-\mathrm{E}_{\mathrm{F}}\right) / \mathrm{k}_{\mathrm{B}} \mathrm{T}} .}
$$

Taking into account only the first excited state, which is the VO-splitting $e$ state with the energy $\mathrm{E}_{\mathrm{vo}}$, and neglecting the higher-lying excited states, $\mathrm{Eq}(3)$ thus can be written as:

$$
\mathrm{n}(\mathrm{T}) \propto \frac{\mathrm{N}}{1+0.5 g_{1} \mathrm{e}^{-\left(\mathrm{E}_{\mathrm{VO}}-\mathrm{E}_{\mathrm{d}}\right) / \mathrm{k}_{\mathrm{B}} \mathrm{T}}+0.5 \mathrm{e}^{\left(\mathrm{E}_{\mathrm{d}}-\mathrm{E}_{\mathrm{F}}\right) / \mathrm{k}_{\mathrm{B}} \mathrm{T}}}
$$

Here $g_{1}$ is the degeneracy factor of the $E_{v o}$ state. $\mathrm{Eq}(4)$ is valid at low temperatures when the donors is mainly in the $E_{d}$ and $E_{v o}$ states. Using $E q(4)$ for fitting the temperature dependence of the donor concentration on the ground state estimated by EPR at low temperatures (below $40 \mathrm{~K}$ in our experiments), we can obtain $g_{1},\left(E_{d}-E_{F}\right)$ and $\left(E_{V O}-E_{d}\right)$, i.e. the VO splitting of the donor.

With increasing further the temperature, electrons from the $E_{v o}$ and $E_{d}$ states can be excited to the higher-lying $2 p$ state. In this temperature range ( $T \geq 40 \mathrm{~K}$ ), $\mathrm{k}_{\mathrm{B}} \mathrm{T} \geq 3.4 \mathrm{meV}$ and is larger than the ( $\left.E_{v o}-E_{d}\right)$ separation of $\sim 3 \mathrm{meV}$. The populations on the $E_{d}$ and $E_{v o}$ are expected to be similar and the decrease of the EPR signal is mainly due to the electron removal from $\mathrm{E}_{\mathrm{d}}$ to the $2 p$ state. Neglecting the $E_{v o}$ state and considering only the $\mathrm{E}_{\mathrm{d}}$ and $2 p$ state, Eq(3) can be written as

$$
\mathrm{n}(\mathrm{T}) \propto \frac{\mathrm{N}}{1+C \mathrm{e}^{-\left(\mathrm{E}_{2 \mathrm{p}}-\mathrm{E}_{\mathrm{d}}\right) / \mathrm{k}_{\mathrm{B}} \mathrm{T}}+0.5 \mathrm{e}^{\left(\mathrm{E}_{\mathrm{d}}-\mathrm{E}_{\mathrm{F}}\right) / \mathrm{k}_{\mathrm{B}} \mathrm{T}} .}
$$

Here the factor $\mathrm{C}$ accounts for the occupation of the higher excited states within $\mathrm{k}_{\mathrm{B}} \mathrm{T}$ from the $2 p$ state. Since the Fermi level $E_{F}$ can change when the excited state is populated so in all the fits, $E_{F}$ is a fitting parameter. However, since the $\mathrm{E}_{\mathrm{VO}}$ level is very close to $\mathrm{E}_{\mathrm{d}}, \mathrm{E}_{\mathrm{F}}$ was found to slightly move up from $\sim 2.7 \mathrm{meV}$ to $\sim 3.8 \mathrm{meV}$ above $\mathrm{E}_{\mathrm{d}}$, i.e. just above the $\mathrm{E}_{\mathrm{vo}}$ level.

\section{References}

[1] N.W. Ashcroft and N.D. Mermin, Solid State Physics (Books/Cole, Bermont, 1976), p. 581. 